\title{
Acute Bronchiolitis-Should We Be Doing More?
}

SIOBHAN B. CARR

Department of Paediatric Respiratory Medicine, Royal Brompton and Harefield NHS Foundation Trust, London

\section{ELEANOR MAIN}

Physiotherapy Department in Respiratory, Critical Care and Anaesthesia, UCL Great Ormond Street Institute of Child Health, London

The mantra of many clinicians when it comes to the inpatient management of infants with acute bronchiolitis is "supportive management and hands off". Recommendations in countries around the globe $^{1,2}$ suggest that the best approach is supportive care alone: support respiratory distress with oxygen (stepping up to CPAP or ventilation if needed), support nutrition/fluid balance and wait for the natural history of this acute viral infection to take its course, which for the commonest pathogen, respiratory syncytial virus is a gradual worsening for 5 days from onset of coryzal symptoms then a slow recovery taking about 3 weeks. They all say do not perform chest physiotherapy in children with no comorbidities. Even in France, one country that has recommended physiotherapy for bronchiolitis, evidence could not be found for improved symptoms or length of stay in a large blinded randomized controlled study using increased exhalation technique physiotherapy with assisted cough in just under 500 infants $^{3}$.

This year's Cochrane review included data from 12 studies in 9 countries across three continents, concluding that routine physiotherapy for hospitalized infants and children under 2 with bronchiolitis could not be recommended because there was a risk of harm and a lack of proven benefit. ${ }^{4}$ In particular the review emphasized that 'forced expiratory techniques' in severe bronchiolitis could be related to adverse effects such as vomiting, bradycardia with desaturation and respiratory destabilization.

A paper in this month's journal ${ }^{5}$ seems to fly in the face of this well-established stance. A large blinded randomized trial of physiotherapy in any paediatric respiratory condition is hard to find, but one in the acute infant condition of bronchiolitis suggesting we step in and perform airway clearance techniques is at odds with common wisdom. However, this study presents evidence for the benefits of two different physiotherapy modalities for infants with bronchiolitis at the milder end of the spectrum. 
Assisted autogenic drainage (AAD) and intrapulmonary percussive ventilation (IPV, a high frequency oscillation delivered in short bursts via a face mask), are known as airway clearance interventions, with IPV potentially having some PEEP effects too. Both claim to recruit the smaller airways where secretions are thought to cause the obstructive pattern seen in bronchiolitis. They might be expected to impact positively on increased mucus production, hyperinflation and atelectasis but would not be expected to improve acute inflammation, edema or bronchospasm. The theoretical rationale of physiotherapy treatment for acute bronchiolitis in infants, to relieve mucus plugging, lung hyperinflation or atelectasis, is credible, but the evidence base has so far failed to materialize. This may be due in part to the inflammation, tachypnea, paroxysmal cough and critical airway narrowing which usually co-exist in this condition and which are exacerbated by these treatments. Physiotherapy for infants with severe bronchiolitis is therefore fraught with balancing clinical benefit against the risk of triggering respiratory failure.

One of the modalities investigated in this study (AAD) arguably has similar characteristics to the 'forced expiration techniques' that attracted concerns in the Cochrane review. During AAD, expiratory flow is manipulated by compression over the chest wall, with a view to increasing flow velocity. The suggestion from this study is that a modification of this same treatment modality in this milder population will result in potentially opposite effects. It is unclear what the mechanism for this effect would be, or how to properly advise physiotherapists in future. The two physiotherapists in the trial were "well trained", these are highly specialized physiotherapy techniques and are unlikely to be available in the average general paediatric ward setting.

This month's paper suggests that these physiotherapy techniques with the help of assisted cough and nasopharyngeal rinsing when required, both reduce hospital stays by up to a day. A reduction in length of stay (LOS) for this common condition by just a day would have massive implications on costs to health services, although there is evidence that LOS is already reducing with just supportive care: in the United States a recent study ${ }^{6}$ showed a mean LOS of 2.4 days compared to studies in the early nineties showing 3.3 days. ${ }^{7}$ The Wang scores in this study were lower than any of those described in the Cochrane studies and days to discharge even in the control group of this study, equivalent to the lowest of those in the Cochrane studies, suggesting these were mildly affected infants. It is unclear how a single daily 20 min treatment would have such a significant effect on length of hospital stay unless it is because of the mild nature of the illness in this group.

Would symptoms so mild justify the time and resources for these treatments? Yes, certainly if the time to discharge can reliably be shortened in this way. What is unclear is whether other factors 
were at play. Were Wang scores or discharge decisions reliable? To what extent might the nasopharygeal rinsing or tracheal stimulation have influenced results?

It remains to be seen whether this study is going to turn into another hypertonic saline debate, lauded as the first treatment to be effective in bronchiolitis; producing one of the first paediatric Cochrane reviews that actually suggested there was enough evidence available to recommend a treatment. ${ }^{8}$ Only later to be shown to have no effect after some further large clinical trials and metaanalysis, $^{9,10}$ the original review may have been caught out by the large heterogeneity of many of the hypertonic saline trials.

As the article itself says: more studies are needed, but well done to the research team presenting the paper on getting this novel study fully recruited to. Now to the harder part: implementation or further studies? Until we know for sure, this study alone should probably not supersede the caution recommended by the Cochrane review on physiotherapy ${ }^{4}$ in this vulnerable patient group.

\section{REFERENCES}

1. Bronchiolitis in children: diagnosis and management. NICE Guidelines (NG9), June 2015; ISBN: 978-1-4731-1162-2.

2. Ralston SL, Lieberthal AS, Meissner HC. Clinical practice guideline: the diagnosis, management, and prevention of bronchiolitis. Pediatrics 2014;134:e1474-e1502.

3. Gajdos V, Katsahian S, Beydon N. Effectiveness of chest physiotherapy in infants hospitalized with acute bronchiolitis: a multicenter, randomized, controlled trial. PLoS Med 2010;7: e1000345.

4. Roque I figuls M, GineGarriga M, Granados Rugeles C. Chest physiotherapy for acute bronchiolitis in paediatric patients between and 24 months old. Cochrane Database Syst Rev 2016;2:CD004873.

5. Ginderdeuren F, Vandenplas Y, Deneyer M. Effectiveness of Airway Clearance techniques in pediatric patients hospitalized with acute bronchiolitis. Ped Pulm 2016.

6. Corneli HM, Zorc JJ, Holukov R, Kuppermann L. Bronchiolitis clinical characteristics associated with hospitalization and length of stay. Pediatr Emerg Care 2012;28:99-103.

7. Pelletier AJ, Mansbach JM, Camargo CAJ. Direct medical costs of bronchiolitis hospitalizations in the United States. Pediatrics 2006;118:2418-2423.

8. Zhang L, Mendoza-Sassi RA, Wainwright C, Klassen TP. Nebulised hpertonic saline solution for acute bronchiolitis in infants. Cochrane Database Syst Rev 2013;31:CD006458. 
9. Brooks CG, Harrison WN, Ralston SL. Association between Hypertonic saline and hospital length of stay in acute viral bronchiolitis: a reanalysis of 2 meta-analyses. JAMA Pediatr 2016;170:577-584.

10. Maguire C, Cantrill H, Hind D, Bradburn M, Everard ML. Hypertonic saline (HS) for acute bronchiolitis: systematic review and meta-analysis. BMC Pulm Med 2015;15:148. 